\title{
Random Raman fiber laser based on a twin-core fiber with FBGs inscribed by femtosecond radiation
}

\author{
M. I. Skvortsov, ${ }^{1,2}$ S. R. Abdullina, ${ }^{1, *}$ A. A. Wolf, ${ }^{1,2}$ A. V. Dostovalov, ${ }^{1,2}$ A. A. Vlasov, ${ }^{1}$ I. A. Lobach, ${ }^{1,2}$ \\ S. WABNITZ, ${ }^{2,3,4}$ (i) AND S. A. BABIN ${ }^{1,2}$
}

${ }^{1}$ Institute of Automation and Electrometry SB RAS, Novosibirsk, Russia

${ }^{2}$ Novosibirsk State University, Novosibirsk, Russia

${ }^{3}$ DIET, Sapienza Università di Roma, via Eudossiana 18, 00184 Rome, Italy

${ }^{4}$ Istituto Nazionale di Ottica del Consiglio Nazionale delle Ricerche (INO-CNR), via Branze 45, 25123 Brescia, Italy

*Corresponding author: abdullina.sofia@gmail.com

Received 31 October 2018; revised 11 December 2018; accepted 12 December 2018; posted 12 December 2018 (Doc. ID 349826);

published 9 January 2019

\begin{abstract}
Narrowband Raman lasing in a polarization-maintaining two-core fiber (TCF) is demonstrated. Femtosecond pointby-point inscription of fiber Bragg gratings (FBGs) in individual cores produces a half-open cavity with random distributed feedback. The laser linewidth in the cavity with a single FBG inscribed in one core of the TCF reduced by $\sim 2$ times with respect to the cavity with a fiber loop mirror. It is shown that the inscription of two FBGs in different cores leads to the formation of a Michelson-type interferometer, leading to the modulation of generation spectra near threshold. This technique offers new possibilities for spectral filtering or multi-wavelength generation. (c) 2019 Optical Society of America
\end{abstract}

https://doi.org/10.1364/OL.44.000295

Raman fiber lasers operating via random distributed feedback (RDFB) are of interest for fundamental studies of nonlinear optical effects in disordered media [1], and have potential application as practical laser devices, owing to their very simple design consisting of just a passive fiber coupled to a pump source $[2,3]$. Amplification and positive feedback, both necessary for lasing, are provided here by stimulated Raman scattering induced by the pump radiation and Rayleigh backscattering (RBS) from naturally present sub-micrometer disorder in silica fibers, respectively. As the RBS-based RDFB is extremely broadband, the RDFB fiber laser is capable to emit in the whole transmission window of silica fibers $(1-2 \mu \mathrm{m})$. Moreover, such a broad wavelength tuning is possible with a single pump source operating around $1 \mu \mathrm{m}$, due to the efficient cascaded generation of higher-order Stokes waves. This is possible in a RDFB laser scheme, even in the absence of resonant cavities for each order [4,5], a condition which is necessary for the cascaded operation of conventional Raman fiber lasers. Even for highorder Stokes waves, the optical efficiency of pump-to-Stokes conversion is as high as $\sim 70 \%$ (for a quantum efficiency $>80 \%$ ) [4], thanks to the absence of intra-cavity elements (and their corresponding insertion losses) in a RDFB fiber laser. The maximum efficiency, defined by light attenuation along the fiber [6], may be reduced by shortening the fiber in combination with a corresponding increase of pump power in order to overcome the larger generation threshold [7-9]. The firstorder Stokes optical efficiency approaches $90 \%$, which is close to the quantum limit ( $95 \%)$, both for polarization-maintaining (PM) [10] and non-PM [11] fibers. (In the last case, a $120 \mathrm{~m}$ long passive fiber with $>200 \mathrm{~W}$ level pumping is used.)

In the absence of wavelength-selective cavity elements, the laser emission linewidth of RDFB fiber lasers is defined by the spectral profile of the Raman gain, which plays the role of the spectral filter. The observed emission line exhibits a typical Schawlow-Townes narrowing near the generation threshold, then starts to broaden with increasing laser power owing to the nonlinear Kerr effect of self-phase modulation (SPM) [12]. When using kilometer-long fibers, the influence of group velocity dispersion also becomes important with a result that power broadening is suppressed in favor of a cubic root dependence of linewidth as a function of nonlinear phase shift. At the same time, the emission linewidth grows nearly proportionally to the Stokes order for cascaded generation, owing to the increasing effect of cross-phase modulation. The resulting RDFB laser linewidth amounts to about 1,2, and $3 \mathrm{~nm}$ for the first, second, and third order, respectively, at a watts power level [4].

The integration of a narrowband spectral filter into the open cavity of a RDFB laser leads to a linewidth reduction, which is nearly proportional to the ratio between the filter bandwidth and the Raman gain bandwidth. The linewidth of a RDFB laser with a fiber Bragg grating (FBG) as a spectral filter amounts to $\sim 0.2 \mathrm{~nm}$ at several watts power (which makes a frequency doubling in nonlinear crystal possible [13]) and may be as low as $0.02 \mathrm{~nm}$ when a narrowband Fabry-Perot filter is used at low powers [14]. Herewith, the use of a tunable filter makes 
it possible to achieve a broad tuning of the generated Stokes wavelength [6], whereas the use of a comb-like filter enables the multi-wavelength operation of RDFB fiber lasers [14-16]. An additional possibility of line narrowing consists in the reduction of power density in the fiber core, thus reducing nonlinear effects. In particular, one can use large-mode-area [17], multicore [18], or tapered [19] passive fibers. For example, in a random laser based on a twin-core PM fiber with a half-open cavity involving a broadband fiber loop mirror (FLM) [18], the linewidth is five times smaller than that for a random laser based on a conventional single-mode fiber (SMF) with similar parameters [10]. The reasons for the line narrowing include not only the weakening of nonlinear effects due to the power density reduction, but also the spectrally selective properties of the twin-core fiber itself (with a quasi-periodic wavelength transmission function). As a result, the linewidth of RDFB lasers using a twin-core fiber in combination with a broadband FLM amounts to about $0.5 \mathrm{~nm}$ at $\sim 7 \mathrm{~W}$ output power [18].

In this Letter, we report on the development of unique spectral filters based on FBGs inscribed in a twin-core fiber and their implementation for random fiber laser linewidth narrowing. The use of the femtosecond laser technology for FBG fabrication $[20,21]$ enables filter formation either in the selected fiber core or in both cores simultaneously. The FBGs inscribed in different cores may be shifted along the fiber against each other, thus forming in the two-core fiber (TCF) with coupled cores a Michelson-type interferometer, which offers new opportunities for controlling the RDFB fiber laser spectrum.

A PM TCF has been developed and manufactured in FORC RAS (Moscow). The radius of the cores is $3 \pm 0.3 \mu \mathrm{m}$, and the average distance between the core centers is $17.1 \pm 0.3 \mu \mathrm{m}$; the propagation loss coefficient at $1.06 \mu \mathrm{m}$ is measured to be $2.2 \mathrm{~dB} / \mathrm{km}$, and the Raman gain coefficient is $0.85-$ $0.95 \mathrm{~W}^{-1} \mathrm{~km}^{-1}$ [18]. The general scheme of the investigated RDFB laser configurations is shown in Fig. 1. A linearly polarized pump radiation at $1050 \mathrm{~nm}$ generated by an $\mathrm{Yb}$-doped fiber laser is injected into the RDFB laser cavity through a WDM coupler made of a PM SMF spliced with one of the PM TCF cores by a Fujikura LZM-100 splicer. A highly reflective FBG (HR FBG1) is inscribed in the other core used as the input cavity mirror for the first tests. For subsequent experiments, an additional HR FBG2 was also inscribed in the pumped core. Femtosecond inscription technology enables the high-precision FBG positioning inside an individual fiber core by means of the visualization of the modification area in the two coordinates perpendicular to the core axis [20]. It is also possible to precisely shift along the fiber axis the FBGs inscribed in different cores of the TCF [21].

The output end of the TCF was cleaved at an angle of $>10^{\circ}$ in order to avoid the Fresnel reflection, thus securing a RDFB

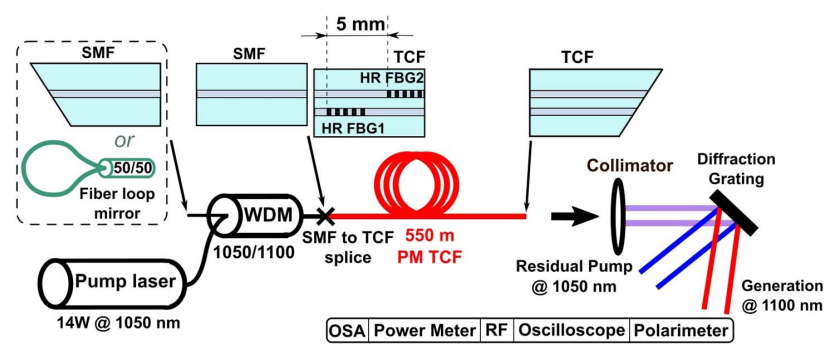

Fig. 1. TCF-based Raman laser with half-open cavity. via Rayleigh backscattering in the half-open cavity with either a single or composite FBG reflector. Note that at the end with FBG(s) either angled cleaving of the WDM output end or its termination by a FLM was explored. The output radiation of the RDFB fiber laser is collimated by a lens (Thorlabs F230FC-B) and dispersed by a diffraction grating (Thorlabs GR25-1210). Four beams are observed at the laser output (generated Stokes and transmitted pump beams from each core).

The generation spectra are characterized by an optical spectrum analyzer (OSA) Yokogawa AQ6370 with a resolution of $20 \mathrm{pm}$. The radio-frequency (RF) spectrum is analyzed by an RF signal analyzer (Agilent N9010A), and the light intensity dynamics is measured by a fast photodetector Thorlabs DET08CF with the oscilloscope Tektronix TDS 3032B. The degree of polarization is determined by polarimeter Thorlabs PAN5710IR2.

At first, we test the configuration with a single HR FBG1 (Fig. 2). Figure 2(a) shows the total (from both cores) power of the generated laser radiation and transmitted pump radiation as a function of the input pump power. The threshold pump power amounts to about $12 \mathrm{~W}$. The generated Stokes power at $1100 \mathrm{~nm}$ grows rapidly above the threshold, reaching $3.4 \mathrm{~W}$ at a maximum pump power of $13 \mathrm{~W}$. The solid lines in Fig. 2 (a) correspond to the results of numerical simulation obtained from the power balance equations, including RDFB due to Rayleigh backscattering, with boundary conditions taken as point-like reflection at the fiber input $\left(R_{l}\right)$ and output $\left(R_{r}\right)$ [22]. The HR FBG1 with reflectivity $\mathrm{R}$ inscribed in one of the TCF cores is equal to a mirror with reflectivity of $R / 2$ due to the strong coupling between the cores, so we used $R_{l}=$ 0.5 in the model. Reflectivity $R_{r}$ is equal to 0 for the half-open cavity configuration with an angled cleaved output fiber end. The Raman gain coefficient is taken to be $0.95 \mathrm{~W}^{-1} \mathrm{~km}^{-1}$, which is within the experimental range for this TCF [18].

The generation spectra for different output powers are shown in Fig. 2(b). The measured linewidth (full width at half-maximum [FWHM]) appears to be by $\sim 2$ times smaller than that for the TCF-based random Raman laser with a FLM [18]. Moreover, the linewidth is weakly dependent on power. Spectral narrowing is caused by the use of a relatively narrowband FBG $(\delta \lambda \approx 400 \mathrm{pm})$ [see Fig. 3(a)], instead of a broadband FLM. Besides, a significant improvement of the generation spectrum stability is obtained when the FBG is used: in Ref. [18], it was noted that several side-lobes on the right wing of the spectrum appear and disappear with a typical
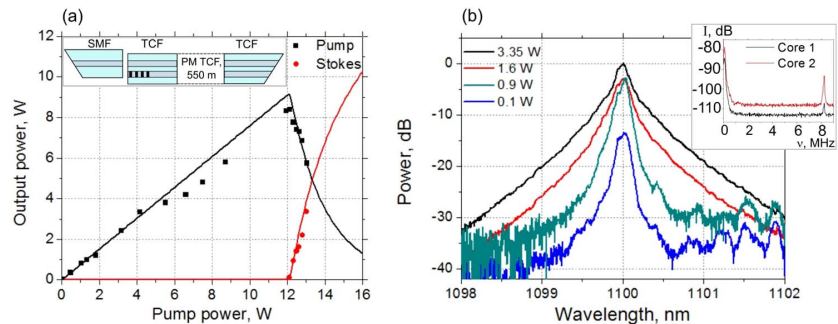

Fig. 2. (a) Output power for transmitted pump (black squares) and generated Stokes (red circles) radiation as a function of the input pump power in the scheme with a single HR FBG1. The solid lines denote a simulation with the balance model. (b) Generation spectra at different levels of output power. RF spectra of output radiation in each core are shown in the inset (spectrum for core 2 is up-shifted by $5 \mathrm{~dB}$ ). 
(a)

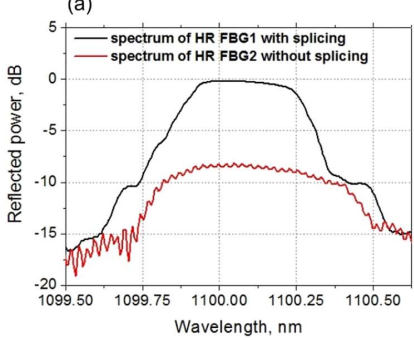

(b)

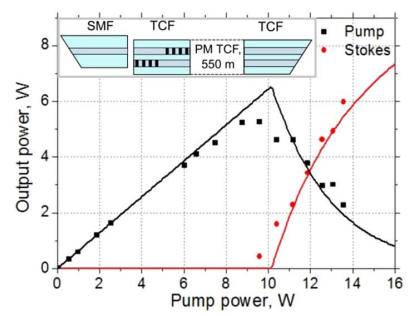

Fig. 3. (a) Measured spectra of HR FBG1 and HR FBG2. (b) Output power of the transmitted pump (black squares) and generated Stokes (red circles) radiation as a function of the input pump power in the scheme with HR FBG1 and HR FBG2 in different cores. The solid lines denote simulations with the balance model.

timescale of about $1 \mathrm{~min}$. We have checked that adding the FLM in our scheme (spliced to the output WDM port; see Fig. 1) does not change the measured spectra significantly, thus proving that FBG reflection is the main stabilizing factor. Figure 2(b) shows residual side-lobes at low powers, but their amplitude (about $-20 \mathrm{~dB}$ ) is now much lower than in Ref. [18]. The inset in Fig. 2(b) shows that the measured RF spectrum of the RDFB laser has no mode structure associated with the $550 \mathrm{~m}$ TCF: only the peaks with a spacing of $\sim 7.5 \mathrm{MHz}$ are seen, which are transferred from the $14 \mathrm{~m}$ long pump laser. The modeless RF spectrum proves the random character of the feedback.

As a next step, we inscribed two HR FBGs in different cores, with a position shift along the fiber which is less than the beating length between the cores $(\sim 5 \mathrm{~cm})$. The spacing between input FBG centers is $\sim 5 \mathrm{~mm}$ at the FBG length of $3 \mathrm{~mm}$. Their reflection spectra are shown in Fig. 3(a): the spectral widths of HR FBG1 and HR FBG2 are $\approx 400$ and $\approx 650 \mathrm{pm}$, respectively. The HR FBG1 spectrum was measured through a SMF spliced to the corresponding TCF core, whereas the HR FBG2 spectrum was obtained without splicing, i.e., with additional losses reducing the spectral amplitude. The shift along the fiber between the FBGs leads to the formation of a Michelson interferometer because of the power transfer between the cores.

The measured generation spectra for the scheme with two input FBGs are shown in Fig. 4. At low Stokes wave power $(\leq 10 \mathrm{~mW})$, a modulation in the spectra is observed [Fig. 4(a)]. The modulation period is about $80 \mathrm{pm}$, which corresponds to the Michelson interferometer with an arm's length difference equal to the distance between FBGs $(5 \mathrm{~mm})$. The width of the individual peaks is below the OSA resolution $(20 \mathrm{pm})$. With increasing generation power, the modulation is vanishing because of the significant broadening of the peaks. The generation linewidth (FWHM) at the output power of $100 \mathrm{~mW}$ is equal to $160 \mathrm{pm}$ [Fig. 4(a)], and it approaches $550 \mathrm{pm}$ at the maximal power of $6 \mathrm{~W}$ [Fig. 4(b)].

In Fig. 5(a), we compare the laser linewidth as a function of output power for different configurations of the TCF-based random laser. In spite of the extremely narrow peak width near the threshold $(\sim 10 \mathrm{~mW})$, the linewidth of a random fiber laser with two input FBGs at powers $>100 \mathrm{~mW}$ is the largest one. In this case, the linewidth rapidly grows with larger powers, until it nearly saturates at the level corresponding to the FBG
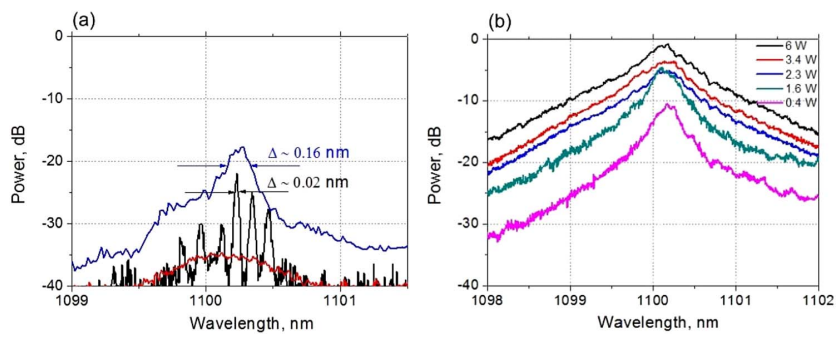

Fig. 4. (a) Output Stokes spectra measured near the generation threshold. (b) Generation spectra measured at different power levels.

bandwidth $(\sim 600 \mathrm{pm})$. At maximum power $(6 \mathrm{~W})$, the linewidth is nearly equal to that of a random laser with a broadband FLM [18]. However, when compared with the FLM-based laser with an unstable spectrum, i.e., exhibiting time-fluctuating side-lobes [18] [see Fig. 5(b)], the spectrum of the FBG-based random laser is stable, with no slowly varying side-lobes [18]. The narrowest and most stable line is obtained for a random laser scheme with a single input FBG: its width varies only slightly between 150 and $200 \mathrm{pm}$ in the power range of 13.5 W, with a tendency to saturation. The measurements also confirmed that the Stokes radiation in all configurations is quasi-continuous and linearly polarized: the measured polarization extinction ratio is below $-20 \mathrm{~dB}$.

The solid lines on Fig. 5(a) are theoretical curves of spectral broadening owing to SPM, which grows as the cubic root of power (for large group velocity dispersion) [4,18]. The blue curve corresponds to the FLM-based random laser, including an additional spectral filter $(\delta \lambda=500 \mathrm{pm})$ that describes the experimental results for a TCF-based laser with FLM (blue filled triangles) [18]. At the same time, it is close to the results of this Letter for the TCF with two FBGs in different cores (red filled circles). A slight correction of the filter width (to $650 \mathrm{pm}$ ) in correspondence with the second FBG bandwidth (red solid curve) makes the agreement slightly better. Relatively large fluctuations of the linewidth measured at different powers may appear due to the influence of the Michelson interferometer formed by two FBGs.

However, the experimental data (black filled squares) for a TCF random laser with a single FBG (of 400 pm bandwidth) are $\sim 2$ times lower than the calculated values (black solid
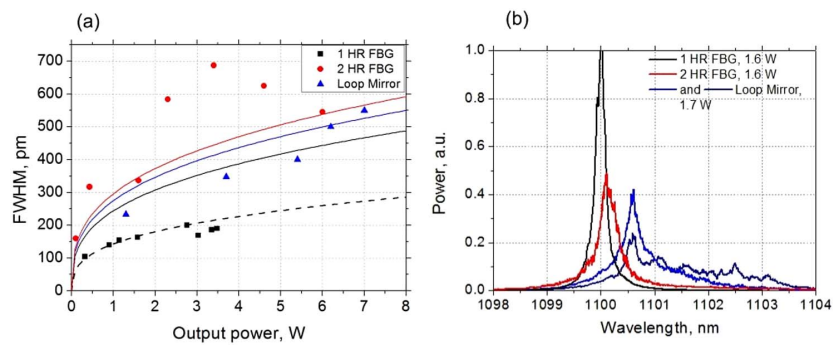

Fig. 5. (a) Spectral width (FWHM) for the generated Stokes radiation for TCF-based random Raman laser configurations with different input reflectors. The solid lines are theoretical fittings at corresponding parameters: $\delta \lambda=650$ (red), 500 (blue), and $400 \mathrm{pm}$ (black); the dashed line corresponds to the configuration with a narrowband filter $(\delta \lambda=80 \mathrm{pm})$. (b) Generation spectra measured at approximately equal output power for different configurations. 
curve). To fit the theory to the experimental data in this case, one should assume a much more narrowband spectral filter (namely, about $80 \mathrm{pm}$ ) than the spectral bandwidth of the input FBG ( 400 pm); see the dashed black curve on Fig. 5(a). Thus, if two nearly equal FBGs terminate both cores of the TCF, the laser linewidth is nearly described by the single-core theory with Raman gain $g_{R}$ и Kerr nonlinearity $\gamma$ coefficients modified for the TCF, correspondingly. However, if one core remains open, an additional filtering effect comes into play. This effect is most probably introduced by the wavelengthdependent transmission through one core of the TCF, as defined by linear coupling among cores, just as for the TCF-based laser with the FLM coupled to one TCF core [18].

In conclusion, random lasing in a twin-core PM fiber with one or two FBGs inscribed by a femtosecond laser in different cores has been demonstrated. Femtosecond technology enables introducing a precise shift between the FBGs along the fiber, thus forming a Michelson-type interferometer within the complex reflector. As a result, multiline generation with the width of individual peaks below the OSA resolution $(20 \mathrm{pm})$ has been observed at the power level of $10 \mathrm{~mW}$. This is similar to the best results obtained when a Fabry-Perot interferometer is introduced inside the open cavity of a random fiber laser [14], but the implementation of our laser is much simpler. The proposed scheme provides the opportunity for multi-wavelength generation with variable spacing or spectral selection of a single narrowband line in a simple scheme, involving two FBGs inscribed in a TCF with variable spacing and bandwidth. In the scheme with a single HR FBG inscribed in one TCF core, narrow line $(<200 \mathrm{pm})$ linearly polarized laser generation has been obtained. The linewidth is much more stable and narrow than that for a TCF-based random laser with a fiber loop mirror [18]. The comparison with theory shows that in the case of a reflector (filter) put at only one core with the other core of the TCF left open, the laser linewidth is much narrower than the calculated values for a conventional SMF with the same reflector that may be explained by additional filtering due to the wavelength-dependent one-core transmission function of the TCF with coupled cores. However, that is not the case for nearly equal reflectors in both the cores when the SMF theory nearly agrees with the experimental data for the TCF with two FBGs at high powers whereas, at low powers, interference between the waves reflected from different FBGs with a shift along the fiber results in additional modulation in the spectrum.

Thus, the combination of random distributed feedback and complex reflector based on refractive index structures inscribed in a multicore fiber opens a new approach for tailoring the output laser spectrum from a single narrow line to a complex multiline spectrum, controlled by the spatial parameters of FBGs inscribed in different cores.

The high-quality narrowband beams generated in multicore fiber random laser configurations may find application in highpower laser devices, multicore fiber sensing and shape measurement techniques, and multicore transmission/amplification of information signals, including multi-wavelength ones, lowcoherence interferometry, and other technologies. The demonstrated line narrowing opportunities of such lasers may be used for efficient frequency doubling and parametric generation, thus increasing the operating wavelength range and potential applications of random fiber lasers.

Funding. Ministry of Education and Science of the Russian Federation (Minobrnauka) (14.Y26.31.0017); Russian Foundation for Basic Research (RFBR) (Russia-Italy 1852-7822)

Acknowledgment. The authors are grateful for the use of equipment from the multiple-access center "High-resolution spectroscopy of gases and condensed matters" at IAE SB RAS, the provision of the twin-core fiber samples by S. L. Semjonov and V. V. Velmiskin, and the technical support by E. A. Evmenova and S. I. Kablukov.

\section{REFERENCES}

1. D. Wiersma, Nat. Phys. 4, 359 (2008).

2. S. K. Turitsyn, S. A. Babin, A. E. El-Taher, P. Harper, D. V. Churkin, S. I. Kablukov, J. D. Ania-Castañón, V. Karalekas, and E. V. Podivilov, Nat. Photonics 4, 231 (2010).

3. S. K. Turitsyn, S. A. Babin, D. V. Churkin, I. D. Vatnik, M. A. Nikulin, S. I. Kablukov, J. D. Ania-Castañón, V. Karalekas, and E. V. Podivilov, Phys. Rep. 542, 133 (2014).

4. S. A. Babin, E. A. Zlobina, S. I. Kablukov, and E. V. Podivilov, Sci. Rep. 6, 22625 (2016).

5. L. Zhang, H. Jiang, X. Yang, W. Pan, S. Cui, and Y. Feng, Sci. Rep. 7, 42611 (2017).

6. S. A. Babin, A. E. El-Taher, P. Harper, E. V. Podivilov, and S. K. Turitsyn, Phys. Rev. A 84, 021805 (2011).

7. I. D. Vatnik, D. V. Churkin, E. V. Podivilov, and S. A. Babin, Laser Phys. Lett. 11, 075101 (2014)

8. H. Zhang, P. Zhou, H. Xiao, and X. Xu, Laser Phys. Lett. 11, 075104 (2014).

9. Z. Wang, H. Wu, M. Fan, L. Zhang, Y. Rao, W. Zhang, and X. Jia, IEEE J. Sel. Top. Quantum Electron. 21, 900506 (2015).

10. E. A. Zlobina, S. I. Kablukov, and S. A. Babin, Opt. Lett. 40, 4074 (2015).

11. X. Du, H. Zhang, X. Wang, P. Zhou, and Z. Liu, Opt. Lett. 41, 571 (2016).

12. D. V. Churkin, I. V. Kolokolov, E. V. Podivilov, I. D. Vatnik, M. A. Nikulin, S. S. Vergeles, I. S. Terekhov, V. V. Lebedev, G. Falkovich, S. A. Babin, and S. K. Turitsyn, Nat. Commun. 2, 6214 (2015).

13. E. I. Dontsova, S. I. Kablukov, I. D. Vatnik, and S. A. Babin, Opt. Lett. 41, 1439 (2016).

14. S. Sugavanam, N. Tarasov, X. Shu, and D. V. Churkin, Opt. Express 21, 16466 (2013).

15. Y. Y. Zhu, W. L. Zhang, and Y. Jiang, IEEE Photonics Technol. Lett. 25, 1559 (2013)

16. A. E. El-Taher, P. Harper, S. A. Babin, D. V. Churkin, E. V. Podivilov, J. D. Ania-Castanon, and S. K. Turitsyn, Opt. Lett. 36, 130 (2011).

17. H. Zhang, L. Huang, P. Zhou, X. Wang, J. Xu, and X. Xu, Opt. Lett. 42, 3347 (2017).

18. A. E. Budarnykh, I. A. Lobach, E. A. Zlobina, V. V. Velmiskin, S. I. Kablukov, S. L. Semjonov, and S. A. Babin, Opt. Lett. 43, 567 (2018).

19. H. Zhang, J. Ye, P. Zhou, X. Wang, J. Leng, J. Xu, J. Wu, and X. Xu, Opt. Lett. 43, 4152 (2018).

20. A. V. Dostovalov, A. A. Wolf, A. V. Parygin, V. E. Zyubin, and S. A. Babin, Opt. Express 24, 16232 (2016).

21. S. S. Yakushin, A. A. Wolf, A. V. Dostovalov, M. I. Skvortsov, S. Wabnitz, and S. A. Babin, Opt. Fiber Technol. 43, 101 (2018).

22. I. D. Vatnik, D. V. Churkin, and S. A. Babin, Opt. Express 20, 28033 (2012). 